Aus der Chirurgischen Universitătsklinik der Charitê in Berlin. (Direktor: Geh.-Rat Hildebrand.)

\title{
Funktionelle Beeinflussung des Gehirns mittels direkt eingespritzter Substanzen.")
}

Von Dr. Franz Breslauer-Schück, Priv.-Doz. für Chirurgie.

Bei meinen Versuchen über Gehirnverletzungen und BewuBtseinsstörung bewährte sich eine Methode, die ich dann systematisch ausarbeitete. Obwohl die Versuche noch nicht abgeschlossen sind, soll hier kurz das Wesentlichste mitgeteilt werden.

Die Oehirnsubstanz läBt sich durch direkte Einspritzung von Flüssigkeiten unmittelbar funktionell beeinflussen, und $z$ war sowohl iu erregendem als auch in lähmendem Sin $\mathrm{ne}$. (Ich betone: Einspritzung in die. Gehirnsubstanz selbst; die Gehirnoberfläche ist bekanntlich nur in einem verhältnismäBig geringen und trotz aller Versuche klinisch nicht verwertbarem MaBe chemisch erregbar.)

Die Technik der direkten Injektion, die an anderer Stelle genauer beschrieben werden soll, ist sehr einfach: die der üblichen Gehirnpunktion. Mittels einer feinelf genügend langen Kanüle beherrscht man von einer kleinen, wenige Millimeter im Durchmesser fassenden Trepanationsöffnung aus das ganze Gehirn.

Die Wirkungen, die sich damit erzielen lassen, sind sehr verschiedenartig; ich will heut, entsprechend der Reihenfolge meiner Versuche, nur von der Lähmung und Erregung des Gehirns sprechen.

$M$ it der Injektion der üblichen $A$ nästhetika (Kokain und seine Ersatzpräparate) $k a n n m a n$ die Leitung $s$ bahnen des Gehirns genau so unterbrechen, wie irgendein peri. pherisches Nervengebiet. Dadurch kann man jede motorische und sensible Bahn in beliebiger Tiefe unterbrechen, das heiBt, jede beliebige Stelledes Gehirns durch Einspritzung der üb. lichen kleinen Dosis für sich allein funktionell aus. schalten. Die örtliche Begrenzung und die Dauer wird, wie bei jeder anderen Anästhesie, durch Zusatz von Adrenalin verstärkt.

Als praktisches Beispiel eine Anwendung am Menschen: Ich habe bei 2 Epileptikern (alte Entlastungstrepanation mit schweren Anfällen vom Jakson-Typ) mehrfach im Beginn der Anfälle wenige Kubikzentimeter $1 \%$ ige Novokainlösung unter die primär gereizten Zentren gespritzt und dadurch die Anfälle sofort unterbrochen. Das ist nach Art der Methode selbstverständlich. Ebenso lassen sich alle anderen therapeutischen Möglichkeiten aus der ein. fachen Ueberlegung herleiten, daB jede Stelle des Gehirns für sich allein ohne Nebenverletzung ausschaltbar ist

$\mathrm{Ob}$ und wie weit solche therapeutischen Versuche von praktischer Wichtigkeit betreffs Dauererfolge sind, kann nur die Erfahrung entscheiden. Ich erwähne die hier angeführten Fälle nur als Beispiel der Anwendbarkeit am Menschen.

In physiologisch-diagnostischer Beziehung bedarf die Methode keiner Empfehlung. Sie ist hier allen andern an schonender Differenzierung weit überlegen. $L u d$ wig vergleicht die experimentellen Hirnversuche mit dem Zergliedern einer Taschenuhr durch Pistolenschüsse. Die direkte Anästhesierung aber arbeitet ohne jede Nebenverletzung und schafft auBerordentlich einfache Versuchsbedingungen.

Als Beispiel für die lokalisatorischen Dienste, die die Methode leisten kann, diene folgendes: Ich hatte bei der Untersuchung von Gehirnverletzungen die Behauptung aufgestellt, da $B$ die BewuBtlosigkeit bei einer großen Reihe von chirurgischen Verletzungen vom Hirnsta $m$ mer ausgelöst wird. Dieser Möglichkeit wurde auf dem diesjährigen Chirurgenkongreß von einer Seite widersprochen. Ich habe daher - und auf diese Weise kam ich zu der gesamten neuen Methode - $2 \mathrm{ccm} 10$ oige Novokainlösung beim Tier in den unteren Teil des Hirnstamms gespritzt. Das Tier war sofort tief bewuBtlos. Die BewuBtlosigkeit hielt etwa $3 / 4$ Stunden an, sie wurde durch Zusatz von Adrenalin $1 / 2$ Stunde verlängert Dieser Beweis hätte sich durch keine andere bisher bekannte Methode mit annähernd derselben Eindeutigkeit erbringen lassen.

Ich will hier von den übrigen theoretisch-diagnostischen Mög: lichkeiten der Methode nicht reden, die sich vielleicht auch für die psychologische Lokalisation verwerten lassen. Arbeiten hierüber sind im Gange.

Es sei noch betont, daß ich nach der Einspritzung von Kokain und Novokain aus feiner Kanüle niemals Erweichungsherde gesehen habe. Beim Tier ist das 8 Tage nach der Einspritzung histologisch nachgeprüft. Beim Menschen konnte, da alle Patienten am Leben blieben, eine histologische Untersuchung nicht erfolgen; doch trat auch nach Wochen keine Funktionsstörung ein, sodaB eine Schä́digung auch hier praktisch ausgeschlossen werden kanin.

Nun zu den Erregungsmitteln: Was in den Leitungsbahnen mit der Anästhesie möglich ist, läßt sich umgekehrt von den Zentren aus mit Erregungsmitteln erreichen. Die Zentren werden isoliert im höchsten Grade in itwer Tätigkeit gesteigert. Ich will hier von. den Tierversuchen mit verschiedenartigen Erregungsmitteln absehen und nur meine ersten vorsichtigen Versuche am Menschen beschreiben. Sie erfolgten mit Koffein. Zusammenfassend läBt sich sagen, daB das von Koffein direkt getroffene Zentrum in seiner

\footnotetext{
1) Nach eirem Vortrag in der Berliner Medizinicehen Oesellschatt am 20. X. 1989.
} 
Funktion auBerordentlich stark gesteigert wird. Die Steigerung ist so stark, daB zu irgendwelchen subjektiven Irrtümern in der Be. urteilung keine Möglichkeit vorhanden ist. lch habe zunächst be alten Kopfschüssen schwer geschädigte und partiell gelähmte motorische Zentren injiziert. Nach der Injektion von Arm- oder Beinzentrum beispielsweise führte der Willensimpuls des Patienten zu einer weit gesteigerten Funktion des betreffenden Gliedes. Es gab Bewegungen her, die vorher der Wille allein nicht erzielen konnte. Diese Wirkung läBt sich bei allen bisher von inir untersuchten Zentren des GroBhirns erzielen. Ein KopfschuB mit schwerer motorischer Sprachstörung erklärte nicht nur selbst nach 5 Minuten spontan, daB er weit besser und leichter sprechen könne als in den letzten Monaten, sondern die Sprache war objektiv für alle Zuhörer deutlich geglättet und beschleunigt. Der Patient brauchte zu bestimmten Wortreihen (zum Beispiel Zählen von 1-10) wesentlich weniger Zeit als vorher. Ein solcher Versuch könnte für sich allein als suggestive Beeinflussung gedeutet werden. Im Rahmen der verschiedenartigen anderen Versuche - insbesondere an den rein motorischen Zentren - beansprucht er Gültigkeit.

Nun zu einem therapeutisch wiclitigen Ergebnis der Koffeinversuche. Es ist das die Wirkung auf das A temzentrum. Ich hatte am Tier festgestellt, daß nach den allerschwersten Gehirnverletzungen (Ausräumung oder Zermalmung des gesamten Gehirns am geöffneten Schädel) das Tier ih Augenblick des Todes durch Einspritzung von Koffein in die Oblongatagegend wieder regelmäBig atmete und noch stundenlang - und bei Wiederholung der Injektion tagelang - am Leben gehalten werden konnte.

Ich habe daher bei verloreneu Patienten kurz vor dem Tode diese Technik angewandt. Nachdem die stärksten intravenösen Wiederbelebungsmittel (groBe Dosen Adrenalin und Koffein intravenös) vergebens gewesen waren, injizierte ich von einer kleinen Trepanationsöffnung am Hinterhaupt aus (es genügt ein Bolırloch von 1-2 mm Durchmesser) 0,75 Koffein in die Gegend der Oblongata. Die Wirkung war durch ihre Stärke überraschend für die Zuschauer. So erlangte ein Patient mit Gehirnembolie, der mit schwerstem Cheyne-Stockeschen Atmen im Sterben lag und alle 2 Mimuten etwa 6mal atmete. und bei dem groBe Dosen Adrenalin und Koffein intravenös versagt hatten, wenige Sekunden nach der direkten Einspritzung in die Gegend der Oblongata eine völlig normale regelmäBige Atmung wieder, die 16 Stunden lang ausreichend durchhielt. Ebenso wurde ein Patient, der nach Operation eines Prostatakarzinoms an schwerer doppelseitiger Pneumonie ad exitum kam, im Augenblick des klinischen Todes injiziert, als die, Atmung aufgehört hatte und der Puls nur noch eben fühlbar war. Wenige Sekunden nach der Einspritzung in die Nähe der Oblongata begann eine tiefe, schnarchende Atmung. Der Patient lebte noch stundenlang und ging erst sekundär zugrunde, als das Herz versagte, wälirend die Atmung bis zum letzten Moment kräftig und regelmäßig war. Da hier wegen des schlechten Herzens eine Wiederholung der Injektion keinen Zweck hatte, wurde sie unterlassen. In allen Fällen dagegen, in welchen das Herz ausreicht und die Todesursache im Atemzentrum liegt, vermag die nach etwa 12 Stunden wiederholte Infiltration der Umgebung des Atemzentrums von neuem den Exitus um viele Stunden herauszuschieben.

Es läBt sich mit Sicherheit sagen, daB die direkte Erregung der Oblongata durch Koffein das stärkste Wiederbelebungs. mittel ist, das wir heut besitzen. Die Indikation seiner Anwendung richtet sich danach, ob eine stunden- und tagelange Erhaltung der Atmung bei der Natur des Leidens lebenserhaltend wirken kann.

Vot1 den Versuchen mit andersartigen Mitteln, die noch nicht abgeschlossen sind, will ich hier noch nichts sagen. Selbstverständlich kommen nicht nur Erregungsmittel bzw. Anästhetika in Frage, sondern auch ganz andersartige Mittel. So habe ich, entsprechend den Bestrebungen der heutigen Zeit, das Großhirn von Tieren mit Hodenextrakt infiltriert. Der Erfolg entsprach den Erwartungen.

Alle hier erwähnten Versuche sind durch Kontrollversuche am Tier mit indifferenten Flüssigkeiten (Kochsalzlösung) auf ihre Eindeutigkeit nachgeprüft.

Ueber die sehr einfache Technik habe ich mich bisher absichtlich nicht ausgesprochen, da für die Ausarbeitung der Methode am Menschen selbstverständlich allergrößte Vorsicht geboten ist. Wie schon gesagt, darf man nur chemisch absolut indifferente Flüssigkeiten benutzen, um Erweichungsherde zu vermeiden. Ich habe solche bei den bisher hier erwähnteu Mitteln nie gesehen.

Blutungen sind bei Vermeidung der Sinus und der basalen Gefäße noch weniger zu befürchten. als bei der gewöhnlichen Gehirnpunktion, weil man zur lnjektion eine besonders feine Nadel nehmen kann. Wohl aber muB man sich bei den Anästhetika vor der Oblongata inachtnehmen; denn jede Anästhesierung der letzteren bringt unweigerlich eine halbstündige Atemlähmung. Ich habe sie beim Tier absichtlich erzeugt (und durch künstliche Atmung überwunden), beim Menschen konnte ich sie durch Anwendung einfacher VorsichtsmaBregeln stets vermeiden:

1. Für die Einspritzung sämtlicher Flüssigkeiten gilt der Grundsatz, daß unter keinen Umständen die Oblongata angestochen oder verletzt werden darf.

2. Bei der Anwendung der Anästhetika sind die Ventrikel und die basalen Liquorräume prinzipiell zu vermeiden, weil durch sie das Anästhetikum zur Oblongata fortgeleitet wird; das heißt also: Vor dem Spritzen ansaugen, wenn Liquor kommt, nicht spritzen und bei Auftreffen auf die Schädelbasis die Nadel (stumpfe) mindestens $1 \mathrm{~cm}$ zurückziehen!

Dieser zweite Satz gilt selbstverständlich wicht für die Exzitantien der Oblongata.

Da auBer der Oblongata im ganzen Gehirn kein vital bedrohter Teil ist habe ich unter Anwendung der genannten VorsichtsmaBregeln bei meinen bisherigen Versuchen am Mensclien nie einen Unfall erlebt. 